\title{
A Sublinear Algorithm for Weakly Approximating Edit Distance
}

\author{
Tuğkan Batu* \\ University of Pennsylvania \\ batu@cis.upenn.edu \\ Avner Magen \\ University of Toronto \\ avner@cs.toronto.edu
}

\author{
Funda Ergün ${ }^{\dagger}$ \\ Case Western Reserve \\ University \\ afe@eecs.cwru.edu \\ Sofya Raskhodnikova \\ MIT \\ sofya@mit.edu \\ Rahul Sami ${ }^{\ddagger}$ \\ Yale University \\ sami@cs.yale.edu
}

\author{
Joe Kilian \\ NEC Laboratories America \\ joe@nec-labs.com \\ Ronitt Rubinfeld \\ NEC Laboratories America \\ ronitt@nec-labs.com
}

\begin{abstract}
We show how to determine whether the edit distance between two given strings is small in sublinear time. Specifically, we present a test which, given two $n$-character strings $A$ and $B$, runs in time $o(n)$ and with high probability returns "CLOSE" if their edit distance is $O\left(n^{\alpha}\right)$, and " $F A R$ " if their edit distance is $\Omega(n)$, where $\alpha$ is a fixed parameter less than 1. Our algorithm for testing the edit distance works by recursively subdividing the strings $A$ and $B$ into smaller substrings and looking for pairs of substrings in $A, B$ with small edit distance. To do this, we query both strings at random places using a special technique for economizing on the samples which does not pick the samples independently and provides better query and overall complexity. As a result, our test runs in time $\tilde{\mathrm{O}}\left(n^{\max \left\{\frac{\alpha}{2}, 2 \alpha-1\right\}}\right)$ for any fixed $\alpha<1$. Our algorithm thus provides a trade-off between accuracy and efficiency that is particularly useful when the input data is very large.

We also show a lower bound of $\Omega\left(n^{\alpha / 2}\right)$ on the query complexity of every algorithm that distinguishes pairs of strings with edit distance at most $n^{\alpha}$ from those with edit distance at least $n / 6$.
\end{abstract}

\footnotetext{
*Supported by ARO DAAD 19-01-1047 and NSF CCR0105337.

${ }^{\dagger}$ Part of this work was done while the author was visiting NEC Laboratories America.

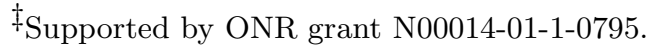

Permission to make digital or hard copies of all or part of this work for personal or classroom use is granted without fee provided that copies are not made or distributed for profit or commercial advantage and that copies bear this notice and the full citation on the first page. To copy otherwise, to republish, to post on servers or to redistribute to lists, requires prior specific permission and/or a fee.

STOC'03, June 9-11, 2003, San Diego, California, USA.

Copyright 2003 ACM 1-58113-674-9/03/0006 ...\$5.00.

\section{Categories and Subject Descriptors}

F.2 [Theory of Computation]: Analysis of algorithms and problem complexity

\section{General Terms}

Algorithms, Theory

\section{Keywords}

String matching, sublinear algorithms, approximation

\section{INTRODUCTION}

Let $A, B$ be two strings over a fixed alphabet $\Sigma$. The edit distance between $A$ and $B$ is defined as the minimum number of character insertions, deletions and substitutions required to transform $A$ to $B$, or vice versa. It ca be verified that this measure provides a symmetric distance. This measure of string similarity is widely used in areas such as computational biology, text processing, and web searching. The edit distance is a well studied measure, and can be computed in slightly under quadratic time [5]. The task of computing or approximating edit distance significantly faster has gained a lot of attention, especially in the bioinformatics community, where the data is very large and and thus fast algorithms are highly desired.

In addition to providing a faster way of estimating the edit distance, a rough approximation algorithm can be used as a preliminary elimination step in conjunction with a slower, more accurate algorithm. In many applications, given many strings, one is interested in computing the edit distance only for pairs of close strings. For string pairs where the distance is above a certain threshold, the actual value of the distance is irrelevant. Thus, a weak approximation algorithm that works in sublinear time can be used as a part of an exact algorithm for quickly distinguishing pairs of strings that are similar from those that are highly dissimilar. The slower, more accurate (or exact) algorithm can then focus on a small 
fraction of the input strings, while the amortized running time over all the given strings stays low.

\section{Our results}

We show that if one is willing to accept a weaker quality of approximation, one can solve the edit distance problem surprisingly quickly. In fact, we give (to our knowledge) the first sublinear time algorithm for approximating the edit distance in the following sense. We show that one can distinguish w.h.p. pairs of strings of length $n$ which have edit distance $n^{\alpha}$ from those which have edit distance $\Omega(n)$ in $\tilde{O}\left(n^{\alpha / 2}\right)$ time for $\alpha \leq 2 / 3$. For any constant $1>\alpha>2 / 3$, our running time is $\tilde{O}\left(n^{2 \alpha-1}\right)$, which is still sublinear. At the core of our algorithm is a sublinear-time procedure which constructs a useful implicit representation of all locations in $A$ at which there are approximate copies of a specific substring of $B$.

Finally, we prove that every algorithm that distinguishes pairs of strings with edit distance $n^{\alpha}$ from strings with edit distance at least $n / 6$ requires $\Omega\left(n^{\alpha / 2}\right)$ queries. To achieve this, we show a lower bound which applies to every algorithm that distinguishes a pair $(A, B)$ of random strings from a pair $\left(A^{\prime}, B^{\prime}\right)$ where $A^{\prime}$ is random and $B^{\prime}$ is a right shift of $A^{\prime}$ by $t$ positions for a random $t \in\left[n^{\alpha} / 2, n^{\alpha}\right]$. This implies the lower bound for the edit distance problem, since two random $n$-bit strings have edit distance at least $n / 6$ with high probability.

\section{Related work}

The edit distance problem is closely related to the longest common subsequence (LCS) and sequence alignment problems: computing the LCS exactly is the same as computing the edit distance. (An analogous statement cannot be made for approximate computations.) The fastest algorithm for computing the LCS of two strings of size $n$, with no assumptions on the strings or their distance, is due to Masek and Paterson [5], and runs in time $O\left(n^{2} / \log n\right) .{ }^{1}$ The computation of LCS becomes most expensive when the common substring is of linear length. A similar problem under a different model is investigated in [3], where the communication complexity of the estimation of the edit and LZ distances (the latter a slightly different measure that involves certain block moves) is considered and a low-distortion embedding of the LZ distance into Hamming distance is given.

There is a significant body of related work on the slightly different problem of approximate string matching, where one would like to find all substrings of the text string of size $n$ which match the pattern string of size $m$ with edit distance at most $k$ (insertions, deletions and substitutions of one character). We mention here a small sample of these works. Landau and Vishkin [4] give an $O(n k)$ algorithm for this problem. Chang and Lawler [1] consider the case when the text string is random and errors are not too frequent. For this case, they give an algorithm which runs in sublinear expected time, namely $O((n / m) k \log m)$ time for $k<m / \log m+O(1)$. Myers [6] improves on their result but requires linear-time preprocessing on one of the two strings. The first subquadratic time algorithm for general approximate string matching was given by Sahinalp and Vishkin [7], running in time $O(n \cdot(1+p o l y(k) \cdot 1 / m \log n))$. Cole and Hariharan [2] improved the running time to $O\left(\left(n k^{4} / m\right)+n+m\right)$.

\footnotetext{
${ }^{1}$ The strings need not be be of different length; we present the equal-length case for simplicity.
}

To put our work in perspective, we note that when the two strings are assumed to be close, dynamic programming restricted to the relevant fraction of the matrix can be applied, saving considerable time and space. In our setting this translates to an immediate $O\left(n^{1+\alpha}\right)$ algorithm. Our algorithm is much more efficient, but not surprisingly uses the idea behind this naive saving as one of its components.

\section{Our techniques}

Our techniques are based on the observation that, if two strings have small edit distance, they will have many almost identical (with small Hamming distance) substrings whose locations in the respective strings are similar. We exploit this property by dividing one of the strings into blocks and determining whether most of these blocks occur in similar locations in the other string. In order to detect substrings that match a block with small Hamming distance, it suffices to randomly sample from both strings. To reduce our query (sample) complexity, we use a procedure that we call a "ruler" that collects a sublinear pool of samples from both strings, and then builds a structure containing all matching pairs of the form (location in $A$, block in $B$ ). In addition, we make use of recursion while subdividing our blocks, which allows us to further improve the complexity of the algorithm. Finally, we "quantize" the locations in the strings generated by the matching process for two substrings; that is, we round the "shifts" between two substrings to a multiple of a suitably-chosen parameter. This results in many fewer cases to consider (and, more importantly, to store) when specifying where a block of one string is located in the other.

\section{Overview of paper}

The rest of this paper is structured as follows: Section 2 defines the edit distance testing problem. In Section 3 we develop a sublinear time algorithm for this problem: In Sections 3.1-3.4, we describe our basic techniques, in Section 3.5 we show how to match the blocks in one string to substrings in the other, and in Section 3.6 we present our main algorithm that uses these techniques to estimate the edit distance. In Section 4, we give a lower bound on the query complexity for this problem.

\section{PRELIMINARIES}

The strings we consider consist of $n$ characters over a fixed alphabet $\Sigma$. We usually denote the input strings by $A, B$. The notation $A[i]$ refers to the $i$ th character of string $A$, and $A[i \ldots j]$ refers to the substring of $A$ delimited by the characters at positions $i$ and $j$.

$\mathcal{D}(A, B)$, the edit distance between two binary strings $A$ and $B$, is the minimal number of character insertions, deletions, or replacements required to generate $B$ from $A$, or vice versa.

For simplicity and without loss of generality, we will assume a binary alphabet $\Sigma=\{0,1\}$ and use the terms "bit" and "character" interchangeably. Our definitions, operations, and techniques involving bit strings extend in the obvious way to character strings.

\section{The edit distance testing problem}

We wish to devise an algorithm to distinguish pairs of $n$ bit strings $A, B$ that are close to each other from pairs that are far from each other in terms of their edit distance. The 
required behavior from this algorithm on input $A, B$, and parameters $\alpha, C, 0<\alpha<1, C>1$, is as follows.

- if $\mathcal{D}(A, B) \leq n^{\alpha}$, output $C L O S E$ with probability at least $2 / 3$.

- if $\mathcal{D}(A, B)>n / C$, output $F A R$ with probability at least $2 / 3$.

The output of is unspecified for $n^{\alpha}<\mathcal{D}(A, B) \leq n / C$. We treat $C$ as a fixed constant and do not analyze the dependence of our algorithm on $C$.

\section{A TEST FOR EDIT DISTANCE}

We now describe a recursive algorithm to check whether the edit distance between two $n$-bit strings $A$ and $B$ is small $\left(\leq n^{\alpha}\right)$ or large $(\Omega(n))$. We arbitrarily designate $A$ to be the reference string, against which $B$ is matched. On the highest level, our algorithm is based on the standard divide and conquer paradigm: $B$ is broken up into substrings, which are recursively matched against $A$. The matching for these local patches is pieced together to form a matching (alignment) for the larger string. However, since it would be too expensive to look at all the subintervals, we randomly sample a small number of them and rely on the statistical properties of these matchings. We then analyze the effect of the statistical uncertainties that arise as a result of the sampling.

We start by discussing the relationship between the edit distance of two strings and the similarity of their substrings.

\subsection{Approximate matchings and coordinated matchings}

A matching of $B$ against $A$ describes how $A$ can be obtained from $B$. In particular, it gives an alignment between the matching portions of $A$ and $B$. Consider how a subinterval $I=B[s \ldots e]$ corresponds to $A$. We may think of $I$ as being matched against a substring $A\left[s^{\prime} \ldots e^{\prime}\right]$; the matching involves a sequence of operations on $A\left[s^{\prime} \ldots e^{\prime}\right]$ that transform it into $I$. In general, $s \neq s^{\prime}$; we refer to the quantity $s^{\prime}-s$ as the shift of $I$. The shift is due to external edits required to match the earlier portions of $A$ and $B$. We refer to the number of edit operations needed to transform $A\left[s^{\prime}, e^{\prime}\right]$ to $I$ as the internal edit distance. ${ }^{2}$ Note that there may be many possible low-edit matchings of $I$ against $A$.

We are interested in matchings in which the internal edit distance is a small fraction of the total number of characters being matched.

Definition 1. An interval $I=B[s \ldots e]$ has a $(t, E)$ (approximate) matching with respect to $A$ if for some interval $A\left[s^{\prime} \ldots e^{\prime}\right], s^{\prime}=s+t$ and $\mathcal{D}\left(A\left[s^{\prime} \ldots e^{\prime}\right], I\right) \leq E$.

If $\mathcal{D}(A, B)$ is small, it is apparent that most subintervals in $B$ will have an approximate matching somewhere in $A$. Further, these matching subintervals must have similar shifts, because a change in the shift value can only arise from an edit (specifically, insert or delete) operation. This leads us to consider coordinated matchings:

\footnotetext{
${ }^{2}$ This is no longer a distance function. The corresponding matching of the internal edit distance is also described in the bioinformatics literature as local alignment.
}

DeFinition 2. Let $\mathcal{I}=\left(I_{1}, \ldots, I_{k}\right)$ be a collection of intervals. We say that $\mathcal{I}$ has a $(t, \sigma, E, D)$-coordinated matching with $A$ if for all but $D$ of the intervals $I_{i} \in \mathcal{I}, I_{i}$ has a $\left(t_{i}, E\right)$-matching with $A$, where $\left|t-t_{i}\right| \leq \sigma$.

We can decompose an interval $I$ of size $S$ into $k$ disjoint contiguous subintervals, $\mathcal{I}=\left(\mathcal{I}_{\infty}, \ldots, \mathcal{I}_{\|}\right)$, each of size $S^{\prime}=$ $S / k$ (we assume that $k \mid S$ ). The existence of a coordinated matching of $\mathcal{I}$ indicates that most of the intervals therein are well matched with similar shifts in $A$. Lemma 1 says that if these subintervals have a coordinated matching with suitable parameters then $I$ has an approximate matching.

Lemma 1. Let $A, I, I_{1}, \ldots, I_{k}, S$ and $S^{\prime}$ be as above. If $\left(I_{1}, \ldots, I_{k}\right)$ has a $\left(t, \sigma, \epsilon S^{\prime}, \delta k\right)$-coordinated matching with $A$, then $I$ has a $(t, \beta S)$-approximate matching with $A$, where

$$
\beta=\left(\frac{2 \sigma}{S^{\prime}}+\epsilon+\delta\right)
$$

Proof. (Sketch) We construct a matching for $I$ by stitching together the matchings for $I_{1}, \ldots, I_{k}$, correcting for gaps, overlaps and unmatching subintervals.

Let $I=B[s \ldots e]$ and $I_{i}=B\left[s_{i} \ldots e_{i}\right]$. If $I_{i}$ has a $\left(t_{i}, \epsilon\right)$ approximate matching, we denote by $I_{i}^{\prime}=A\left[s_{i}^{\prime} \ldots e_{i}^{\prime}\right]$ the substring of $A$ that is transformed into $I_{i}$ (choosing arbitrarily if there are multiple matches). $I_{i}^{\prime}$ can be transformed into $I_{i}$ using $\epsilon S^{\prime}$ edit operations. If $I_{i}$ does not have a $\left(t_{i}, \epsilon\right)$ matching (for $t_{i} \in[t-\sigma, t+\sigma]$ ), we define $t_{i}=t$ and $I_{i}^{\prime}$ to be $A\left[s_{i}+t \ldots e_{i}+t\right]$, i.e., the region obtained by translating the interval $\left[s_{i} \ldots e_{i}\right]$ by $t$. We can trivially transform $I_{i}^{\prime}$ to $I_{i}$ using $S^{\prime}$ edit operations.

We transform the interval $A\left[s+t \ldots e_{k}^{\prime}\right]$ into $I$ as follows. If $s_{1}^{\prime}=s+t$, then we simply transform $I_{1}^{\prime}$ into $I_{1}$ using the same edit operations as for that matching. If $s_{1}^{\prime}<s+t$, then the $s+t-s_{1}^{\prime}$ first characters of $I^{\prime}$ are missing from $A\left[s+t \ldots e_{k}\right]$; we add these to the beginning of $A\left[s+t \ldots e_{k}^{\prime}\right]$ using $s+t-s_{1}^{\prime}$ insert operations, and then proceed as before. Similarly, if $s_{1}^{\prime}>s+t$, we trim the first $s_{1}^{\prime}-s-t$ characters from $A\left[s+t \ldots e_{k}^{\prime}\right]$ and proceed as before. We are left with the remaining portion of $A\left[s+t \ldots e_{k}^{\prime}\right]\left(A\left[e_{1}^{\prime}+1 \ldots e_{k}^{\prime}\right]\right)$, which must be transformed into the remaining portion of $I$ $\left(B\left[e_{1}+1 \ldots e_{k}\right]\right)$.

To complete the transformation, we transform $I_{i}^{\prime}$ into $I_{i}$, for $i=2, \ldots, k$, in the same manner, yielding $I$. At each stage, we trim or add to the remaining string so that $I_{i}^{\prime}$ is a prefix, and then perform the transformation from $I_{i}^{\prime}$ to $I_{i}$.

It remains to compute the number of edits required by this transformation. The number of edits required to transform $I_{i}^{\prime}$ to $I_{i}$, for all $i$ for which there are is an approximate match, is at most $\epsilon S^{\prime} k$. For at most $\delta k$ intervals, $I_{i}^{\prime}$ and $I_{i}$ don't have a good match; the trivial transformation costs at most $S^{\prime} \cdot \delta k=\delta S^{\prime} k$.

We must also account for the $\left|s_{i}^{\prime}-(s+t)\right|$ edit operations required prior to transforming $I_{1}^{\prime}$ and the $\left|s_{i}^{\prime}-\left(e_{i-1}^{\prime}\right)+1\right|$ editing operations required to align the remaining string prior to transforming $I_{i}$, for $i>1$. By the definition of a coordinated matching, $\left|s_{i}^{\prime}-(s+t)\right| \leq \sigma$. Since $s_{i}^{\prime}=s_{i}+t_{i}$ we can write,

$$
\begin{aligned}
\mid s_{i}^{\prime} & -\left(e_{i-1}^{\prime}+1\right) \mid \\
& =\left|\left(s_{i}+t_{i}\right)-\left(s_{i-1}^{\prime}+\left(e_{i-1}^{\prime}-s_{i-1}^{\prime}\right)+1\right)\right| \\
& =\left|\left(s_{i}+t_{i}\right)-\left(s_{i-1}+t_{i-1}+\left(e_{i-1}^{\prime}-s_{i-1}^{\prime}\right)+1\right)\right| \\
& \leq\left|t_{i}-t_{i-1}\right|+\left|\left(e_{i-1}^{\prime}-s_{i-1}^{\prime}+1\right)-\left(s_{i}-s_{i-1}\right)\right| .
\end{aligned}
$$


It follows from the definition of a coordinated matching that $\left|t_{i}-t_{i-1}\right| \leq 2 \sigma$. The latter term is simply the absolute difference between the length of $I_{i-1}\left(s_{i}-s_{i-1}\right)$ and the length of $\left|I_{i-1}^{\prime}\right|\left(e_{i-1}^{\prime}-s_{i-1}^{\prime}+1\right)$. If $I_{i-1}^{\prime}$ can be transformed into $I_{i-1}$ using $\epsilon S^{\prime}$ edit operations, this difference cannot be more than $\epsilon S^{\prime}$, and if no such matching exists, then $I_{i-1}^{\prime}$ will have the same length as $I_{i-1}$ by definition. Thus, at most $2 \sigma+\epsilon S^{\prime}$ operation are required per interval, giving at most $2 \sigma k+\epsilon S^{\prime} k$ operations in all.

Recalling that $S=S^{\prime} k$, we have at most $\left(\frac{2 \sigma}{S^{\prime}}+\epsilon+\delta\right) S$ edits required, implying the lemma.

Lemma 2 shows that if a good matching for an interval exists then there must be a coordinated matching of its subintervals.

Lemma 2. Let $A, I, \mathcal{I}=\left(I_{1}, \ldots, I_{k}\right), S$ and $S^{\prime}$ be defined as above. Let $c>1$ and $S>c E$. If $I$ has a $(t, E)$-matching with $A$ then $\mathcal{I}$ has a $(t, E, c E / k, k / c)$-coordinated matching with $A$.

Proof. (Sketch) Let $I=B[s \ldots e]$. We consider the matching from $A[s+t \ldots q]$ to $I$ that has edit distance $E$. For each $I_{i}$, we consider the smallest interval $I_{i}^{\prime}$ of $A$ containing all the characters that are matched to characters in $I_{i}$. If no such characters exist, we do not assign $I_{i}$. We claim that this correspondence induces a $(t, E, c E / k, k / c)$-coordinated matching.

First, we note that the $I_{i}^{\prime}$ s are disjoint, since our edit operations do not change the ordering of the characters of $A$ that are matched. The edit operations that transform $A[s+t \ldots q]$ into $I$ also transform each $I_{i}^{\prime}$ into $I_{i}$. Each edit operation affects only one $\left(I_{i}^{\prime}, I_{i}\right)$ pair or unassigned $I_{i}$ - either it deletes a character from at most one $I_{i}^{\prime}$ or it inserts a character into exactly one $I_{i}$. Hence, the sum of edit distances between $I_{i}^{\prime}$ and $I$ along with the sum of edits assigned to unassigned $I_{i}$ is at most $E$. If more than $k / c$ had edit distance greater than $c E / k$, or were unassigned (with an edit cost of $S^{\prime}>c E / k$ ), this would cause the sum to be greater than $E$, a contradiction.

It remains to show that the translation (shift) $t_{i}$ between each $I_{i}^{\prime}$ and $I_{i}$ satisfies $\left|t_{i}-t\right| \leq E$. Now, consider what happens if we edit $A[s+t \ldots q]$ to obtain $I$, first by deleting the unmatched characters, one by one, and then inserting the new characters, one by one. At each step, we can recompute the matchings and hence the shifts for each $\left(I_{i}^{\prime}, I_{i}\right)$ pair. Each operation can change any $t_{i}$ by at most 1 each way. However, at the end of this process, when $A[s+t \ldots q]=I$, $t_{i}=t$, so the original values of $t_{i}$ could not be more than $E$ away from $t$.

A special case of this lemma is the existence of coordinated matchings where the intervals have no internal edit distance at all.

Lemma 3. Let $A, I, I_{1}, \ldots, I_{k}, S$ and $S^{\prime}$ be as above. If $I$ has a $(t, E)$-matching with $A$, and $k \geq E$, then $\left(I_{1}, \ldots, I_{k}\right)$ has a $(t, E, 0, E)$-coordinated matching with $A$.

We use Lemmata 1 and 2 in concert to detect good matchings. Suppose string $A$ and $B$ have a good matching. We break $B$ up into subintervals and use Lemma 2 to argue that these intervals have a good coordinated matching. In the next section we show how to efficiently detect a good coordinated matching. Once a coordinated matching is detected, we use Lemma 1 to infer the existence of a good matching between $A$ and $B$. There might be a better matching than the inferred one, but what we get is sufficiently good to distinguish between the two cases we consider.

To obtain the strongest result, we apply this technique recursively, taking care that the degradation in the guarantee does not grow too large.

\subsection{Detecting coordinated matchings via sam- pling}

Our algorithm aims to detect a coordinated matching with a very few queries. Given a set of intervals $\mathcal{I}=\left(I_{1}, \ldots, I_{k}\right)$, we would like to determine for which $t, \mathcal{I}$ has a $(t, \sigma, E, D)$ coordinated matching. We actually accomplish an approximate version of this task: For all $t$, if $\mathcal{I}$ has a $(t, \sigma, E, D)$ coordinated matching we detect with high probability that it has a $(t, \sigma, E, D+\epsilon k)$-coordinated matching, for any constant $\epsilon>0$.

In designing our detection routine, COORD-MATCHES, assume for now that we have a subroutine, MATChes $(A, I, E)$, that determines for which $t, I$ has a $(t, E)$-matching with $A$. We will later implement MATCHES recursively using COORDMATCHES. Our actual subroutines only approximate this behavior; we later adapt our technique to accommodate this approximation.

A crucial observation is that a randomly selected set of $O(\log n)$ subintervals will approximate the behavior of the entire set. We use the following simple consequence of the Chernoff bound.

Lemma 4. For any positive $\epsilon$ and $c$, there exists $d$ such that the following is true. Suppose that a randomly chosen element of a set $S$ (such that $|S|=n$ ) has some property $Z$ with probability $p$. If we uniformly sample (with replacement) $d \log n$ elements from $S$, the fraction $p^{\prime}$ of these samples with property $Z$ satisfies $p-\epsilon / 2 \leq p^{\prime} \leq p+\epsilon / 2$ with probability $1-1 / n^{c}$.

We give the sampling procedure COORD-MATCHES in Figure 1. The parameters $A, \mathcal{I}$ and $\sigma$ are as in Definition 2. The parameters $\epsilon$ and $c$ control the accuracy and reliability of the estimate, as analyzed in Lemma 5.

LEMMA 5. With probability $1-1 / n^{c-1}$ over the random coins of COORD-MATCHES, the output $T$ of COORD-MATCHES $(A, \mathcal{I}, \sigma, E, D, \epsilon, c)$ has the following two properties:

1. If $\mathcal{I}$ has a $(t, \sigma, E, D)$-coordinated matching then $t \in T$.

2. If $t \in T$ then $\mathcal{I}$ has a $(t, \sigma, E, D+\epsilon k)$-coordinated matching.

Proof. For any $t$, if at most $D$ intervals $I_{i}$ do not have a $\left(t_{i}, E\right)$-matching where $\left|t_{i}-t\right| \leq \sigma$ then by Lemma 4 , with probability at least $1-1 / n^{c}$ at most a $D / k+\epsilon / 2$ fraction of the $I_{i_{j}}$ s do not have such a matching, in which case $t \in T$. Similarly, if more than $D+\epsilon k$ intervals do not have a $\left(t_{i}, E\right)$ matching with $\left|t_{i}-t\right| \leq \sigma$, then with probability at least $1-1 / n^{c}$ at least a $(D / k+\epsilon)-\epsilon / 2$ fraction of the $I_{i_{j}} \mathrm{~s}$ do not have such a matching, in which case $t \notin T$. Thus, for both types of errors, the probability of making a mistake is thus at most $1 / n^{c}$. Since there are at most $n$ possible errors possible (for each $t$ the number of non-matches can be either too big or too small, but not both), the lemma follows from the union bound. 
COORD-MATCheS $(A, \mathcal{I}, \sigma, E, D, \epsilon, c)$

1. Let $d$ be as in Lemma 4 for the given $\epsilon$ and $c$, and $l=d \log n$. Choose $i_{1}, \ldots, i_{l}$ uniformly and independently from $[1 \ldots k]$.

2. For each $I_{i_{j}}$, compute $T_{j}=\operatorname{Matches}\left(A, I_{i_{j}}, E\right)$.

3. Return $T=\operatorname{Merge}\left(T_{1}, \ldots, T_{l}, \sigma, \Delta\right)$, where $\Delta=(D / k+\epsilon / 2) l$ and MERGE is defined below.

$\operatorname{MERGE}\left(T_{1}, \ldots, T_{l}, \sigma, \Delta\right)$

1. Return the set $T$, where $t \in T$ iff $T_{j} \cap[t-\sigma \ldots t+\sigma]=\emptyset$ for at most $\Delta$ sets $T_{j}$.

Figure 1: Sampling algorithm for (approximately) finding coordinated matches.

\subsection{Quantizing shifts}

Our MATCHES and COORD-MATCHES algorithms may conceivably give an output set $T$ consisting of $n$ elements. While not affecting the query complexity, this by itself is more time than we wish to take. Further, observe that for detecting strings with the edit distance of at most $n^{\alpha}$, we may restrict the allowed shifts to $\left[-n^{\alpha} \ldots+n^{\alpha}\right]$. However, to achieve a $o\left(n^{\alpha}\right)$ running time, we must further restrict the set of possible outputs. We do this by specifying a quantization parameter, $Q$, which governs the precision of the output.

Definition 3. $A Q$-quantization of $t$ is a value $t_{Q}$ such that $t_{Q}=k Q$ for some integer $k$ and $\left|t-t_{Q}\right|<Q$. A set $S_{Q}$ is a $Q$-quantization of a set $S$ if for every $t \in S$, $t$ has a $Q$ quantization $t_{Q} \in S_{Q}$. If an interval has a $(t, E)$-matching with $A$, we say that it has a $\left(t_{Q}, E\right)$-quantized matching with $A$. We say that a set of intervals, $\mathcal{I}$ has a $(t, \sigma, E, D)$ quantized coordinated matching with $A$ if $Q \mid t$ and for all but $D$ of the intervals $I_{i} \in \mathcal{I}, I_{i}$ has a $\left(t_{i}, E\right)$-quantized matching with $A$, where $\left|t-t_{i}\right| \leq \sigma$. We denote by $t[Q]$ the unique value $k Q$ satisfying $-Q / 2<t-k Q \leq Q / 2$, and by $S[Q]$ the minimal set containing $t[Q]$ for every $t \in S$.

Thus, if $t=Q k$, it has a single quantization, $Q k$, and if $Q(k-1)<t<Q k$, then $t$ has two quantizations, $Q(k-1)$ and $Q k$. When we explicitly quantize a value $t$, we choose its closest quantization, $t[Q]$. However, at times rounding errors may cause us to instead obtain the other possible quantization.

The key observation to make is that coordinated matchings already allow for some "wiggle room," in the shifts allowed for the intervals. Adding moderate amounts of quantization doesn't change this wiggle room significantly. Proposition 1 quantifies this relationship; its proof follows immediately from the fact that $t[Q]$ differs from $t$ by at most $Q / 2$ and any quantization $t_{Q}$ differs from $t$ by at most $Q$.

Proposition 1. Let $\mathcal{I}$ be a family of intervals. If $\mathcal{I}$ has $a(t, \sigma, E, D)$-coordinated matching with respect to $A$ then it has a $(t[Q], \sigma+Q / 2, E, D)$-quantized coordinated matching with $A$. If $\mathcal{I}$ has a $(t, \sigma, E, D)$-quantized coordinated matching with $A$ then it has a $(t, \sigma+Q, E, D)$-coordinated matching with $A$.

Intuitively, if we don't make the quantization factor too large then we can make qualitatively the same inferences using quantized shifts as we can using unquantized shifts.

\subsection{Recursively using coordinated matches}

Our COORD-MATCHES algorithm makes calls to MATCHES, which has to find good matches for individual intervals; we now describe how the MATCHES procedure is implemented. Using Lemma 1, we can detect a good match for an interval $I$ by breaking $I$ into subintervals, detecting good coordinated matchings for these intervals, and inferring the existence of good matches for $I$. That is, we call COORD-MATCHES using a suitable decomposition of $I$ and using suitable error tolerances. While running COORD-MATCHES, we make calls to MATCHES on a subset of these subintervals, which are approximated via a call to COORD-MATCHES on a suitable decomposition of these subintervals, and so on. This process yields a multi-stage algorithm in which matches found in a given stage are used recursively to generate matches in the earlier stage.

At each stage, we match smaller intervals, and require that the matches have smaller internal edit distances. Eventually, we seek $(t, E)$ matches in which $E<1$ (hence, $E$ might as well be 0$)$. But note that if an interval $I$ has a $(t, 0)$-matching with respect to $A$, then $A$ must contain interval $I$ unchanged except for a translation or shift by $t$ positions. In this case, we compute the set of allowable $t$ values directly, using the algorithm SHIFTs described below; this forms the final stage of the recursion. We now turn to the description of this algorithm.

\subsection{Finding approximate block shifts using the ruler procedure}

This subsection describes an algorithm to efficiently find substrings in $A$ that match a block (interval) in $B$ approximately. This procedure is at the core of our edit distance testing algorithm.

The approximate matching problem is as follows. Given a block $I=B[s \ldots e]$ of length $b=e-s+1$ in $B$; and a constant $c_{2}>1$, find all indices $s^{\prime}$ such that $A\left[s^{\prime} \ldots\left(s^{\prime}+\right.\right.$ $b-1)$ ] matches $I$, in the sense that the two substrings have Hamming-distance at most $b / c_{2}$. Note that, if $\mathcal{D}(A, B)<$ $n^{\alpha}$, it is enough to consider $s^{\prime} \in\left[s-n^{\alpha}, s+n^{\alpha}\right]$.

Thus, we now need to solve the following. Given a string $I$ of length $b$, and a string $A^{\prime}=A\left[\left(s-n^{\alpha}\right) \ldots\left(s+n^{\alpha}+\right.\right.$ $b-1)]$, we want to find all shifts $t$ of $I$ within $A^{\prime}$, such that $A^{\prime}[t+1 \ldots t+b]$ matches $I$. That is, we want to find all length $b$ substrings of $A^{\prime}$ with Hamming distance at most $b / c_{2}$ from $I$. Naively, we can randomly sample $O(\log n)$ indices $i$ to determine (with high probability) if the substring $A^{\prime}[(t+1) \ldots(t+b)]$ matches $I$, for a given $t$, and try all $2 n^{\alpha}$ possible shifts $t$. This requires $\Omega\left(n^{\alpha}\right)$ queries to $A$. Below we reduce the number of queries by a "ruler" procedure.

Suppose we would like to compare pairs of characters $A^{\prime}[i], I[j]$ such that some pairs $A^{\prime}[i], I[j]$ are checked for every $i-j$ from 0 to $u=2 n^{\alpha}$. Here is how to achieve this with $\sqrt{u}$ queries to each string, provided that $b>>$ 
$\sqrt{u}$ : In $A^{\prime}$, character positions divisible by $\sqrt{u}$ are queried: $A^{\prime}[\sqrt{u}], A^{\prime}[2 \sqrt{u}], \ldots, A^{\prime}[u]$. In $I, \sqrt{u}$ consecutive positions are queried: $I[1 \ldots \sqrt{u}]$. Intuitively, queries to $A^{\prime}$ act as "centimeter" marks on the ruler, and queries to $I$ act as "millimeter" marks. For every $t=0,1, \ldots, u$, there is a pair of queried positions $A^{\prime}[i], I[j]$ with $i-j=t$. Let cen $=\lfloor t / \sqrt{u}\rfloor$ and $\operatorname{mil}=t \bmod \sqrt{u}$. Then $A[$ cen $\cdot \sqrt{u}]$ and $B[\sqrt{u}-$ mil $]$ are queried positions exactly distance $t$ apart.

We can extend this idea to test whether the entire block matches with shift $l$, using the random sampling idea mentioned earlier: Pick $l=\Theta(\log n)$ numbers $m_{1}, m_{2}, \ldots, m_{l}$ randomly from the range $[0, b-\sqrt{u}]$. For each tick mark on the ruler, construct a fingerprint by querying at $l$ offsets instead of just 1 ; for example, in $A^{\prime}$, the fingerprint of the centimeter mark $\sqrt{u}$ is the sequence of $l$ bits

$$
\left(A^{\prime}\left[\sqrt{u}+m_{1}\right], A^{\prime}\left[\sqrt{u}+m_{2}\right], \ldots, A^{\prime}\left[\sqrt{u}+m_{l}\right]\right) .
$$

Now, we can detect with high probability whether the block matches with shift $t$ by comparing the fingerprints of cen and mil as defined above.

Up to this point we have assumed $b>>\sqrt{u}$. We use the same idea when $b \leq \sqrt{u}$; the only difference is that we need to make the ruler asymmetric. In this case, we can have only $O(b)$ millimeter tick marks, and so we need $\Omega(u / b)$ centimeter tick marks. Thus, in general we can find all matching shifts $l$ by using $O(\max \{\sqrt{u}, u / b\} \log n)$ queries.

\section{Efficient implementation of the ruler}

We now describe a data structure that allows us to efficiently execute the ruler procedure. Recall that we want to detect when a tick mark $i$ in $A^{\prime}$ has the same fingerprint as a tick mark $j$ in $I$. To do this, we maintain a binary search tree, with a leaf corresponding to each fingerprint $f$ encountered thus far. (In practice, a hash tree may be better, but it does not change the asymptotic performance.) Each leaf contains pointers to two linked lists: the $A$-list contains indices $i$ (in $A^{\prime}$ ) that resulted in fingerprint $f$, and the $B$-list contains indices $j$ (in $I$ ) that yielded $f$. It takes $O(\log n)$ time per tick mark, and thus $O(\max \{\sqrt{u}, u / b\} \log n)$ time overall, to build up this data structure.

When all tick marks have been processed, the data structure contains an implicit representation of all shifts $t$ such that $I$ matches $A^{\prime}[t+1 \ldots t+b]$, in the following sense: for each fingerprint $f$, every combination of an index $i$ from $f$ 's $A$-list and $j$ from $f$ 's $B$-list describe a matching shift $t=j-i$. However, it is still potentially expensive to go from this to an explicit list of all matching $t$ values. The problem is simply that there may be $\Omega(u)$ such values.

If we need to know each individual $t$ value precisely, there is no way to avoid this problem. To get around this, the algorithm described in Section 3.6 only uses quantized shift values, i.e., values of $t$ rounded to multiples of some integer $Q$. Reporting distinct multiples of $Q$ for which some $t$ matched, reduces the worst-case size of the output list to $\Omega(u / Q)$. It is easy to take advantage of this reduction with our data-structure: first replace the $B$-list by its $Q / 4$ quantization, $B[Q / 4]$, and similarly replace $A$ by $A[Q / 4]$ (eliminating duplicates in each list). We compute all values $j^{\prime}-i^{\prime}$ for $i^{\prime} \in A[Q / 4]$ and $j^{\prime} \in B[Q / 4]$, and output the set of $Q$-quantized values, $t^{\prime}=\left(j^{\prime}-i^{\prime}\right)[Q]$, eliminating duplicates.

We observe that the resulting rounding errors do not grow too large, and hence that for any $t$ that would have been output in the unquantized case, a $Q$-quantization of $t$ is output by the above algorithm. Let $t=j-i, i^{\prime}=i[Q / 4]$, $j^{\prime}=j[Q / 4]$ and $t^{\prime}=\left(j^{\prime}-i^{\prime}\right)[Q]$. We have $\left|i^{\prime}-i\right| \leq Q / 8$ and $\left|j^{\prime}-j\right| \leq Q / 8$, implying that $\left|\left(j^{\prime}-i^{\prime}\right)-(j-i)\right| \leq Q / 4$. We also have that $\left|\left(j^{\prime}-i^{\prime}\right)[Q]-\left(j^{\prime}-i^{\prime}\right)\right| \leq Q / 2$, from which it follows that

$$
\left|t^{\prime}-t\right|=\left|\left(j^{\prime}-i^{\prime}\right)[Q]-(j-i)\right| \leq 3 Q / 4<Q .
$$

Thus, $t^{\prime}$ is a $Q$-quantization of $t$.

The final algorithm is shown in Figure 2. The following theorem summarizes the performance of this algorithm:

TheOREM 1. Procedure SHIFTs finds all quantized shifts $t$ of interval $I$ in $A^{\prime}$, with high probability. It runs in time $O(\max \{\sqrt{u}, u / b, u / Q\} \log n)$, where $u=\left|A^{\prime}\right|-b$.

Proof. If $t$ is a shift corresponding to an exact match, then the preceding discussion of the ruler shows that the corresponding quantized shift value will be found. If $t$ is a shift corresponding to a Hamming distance of greater than $2 b / c_{2}$, then at least $b / c_{2}$ of the Hamming errors must occur after the first $g$ characters of $I$. Hence, any one $m_{i}$ will find a mismatch with probability at least $1 / c_{2}$. Setting $d=2$, the probability of the fingerprints matching for $t$ is then at most $\frac{1}{n^{2}}$. There are less than $n$ possible shift values, and so the probability of finding any incorrect shift $t$ is at most $\frac{1}{n}$. Higher values of $d$ can be used to obtain error bounds of at most $\frac{1}{n^{c}}$ for any constant $c$, hence the high probability result. The running time bound follows by taking the sum of the time to construct the implicit representation of all shifts, and the time to produce the output.

\subsection{The edit distance testing algorithm}

We now have all the tools we need to build our algorithm for testing edit distance. The algorithm is shown in Figure 3. The top-level procedure is a routine DECIDE that takes as input the two strings $A$ and $B$, and the parameter $\alpha$. DECIDE calls MATCHEs to search for a match of $B$ in $A$ with edit distance at most $n^{\alpha}$; if such a match is found, CLOSE is output, otherwise $F A R$ is output. MATCHES is a recursive procedure, recursing through the procedure COORD-MATCHES. The recursion terminates when the required internal edit distance in each block is less than 1; in this case, MATCHES uses SHIFTS to directly find the matches.

\section{Depth of recursion:}

At each level of the recursive decomposition, the size of the interval input to MATCHES goes down by a factor of $\Omega\left(n^{\alpha-1}\right)$. Thus for any constant $\alpha<1$, there is a constant number $r$ of levels of recursion required to reach a state in which the intervals have size $O\left(n^{1-\alpha}\right)$; at this point, $E<1$ and hence SHIFTs will be called, terminating the recursion.

We assign a height to each call to procedure MATCHES as follows: the final invocation that calls SHIFTs has height 0 , the level above that has height 1 , and so on till we get that the height of the top-level invocation of MATCHES is $r$. We also define the height of an invocation of COORDMATCHES to be the height of the MATCHES procedure that invoked it.

\section{Correctness of the algorithm}

We need to show that with a suitable choice of constants $c_{1}$ and $\epsilon$ (perhaps dependent on $\alpha$ ), procedure DECIDE correctly solves the edit distance testing problem. We first prove that if $\mathcal{D}(A, B) \leq n^{\alpha}$, the algorithm outputs CLOSE. 
$\operatorname{SHIFTs}\left(A^{\prime}, I, Q, c_{2}\right)$

${ }^{*}$ Find all shifts of $I$ in $A^{\prime}$ with Hamming distance $<2|I| / c_{2}$, quantized in multiples of $Q * /$

1. Let $b=|I|, u=\left|A^{\prime}\right|-b$, and $g=\min \left\{b / c_{2}, \sqrt{u}\right\}$.

2. Let $l=d \cdot \log n /\left(-\log \left(1-1 / c_{2}\right)\right)$, for some constant $d>2$. Choose integers $m_{1}, m_{2}, \ldots, m_{l}$ independently and uniformly at random in $[0, b-g]$.

3. Initialize the fingerprint search tree.

4. For $i=g, 2 g, \ldots, u$ do

- Compute fingerprint $f(i)=\left(A^{\prime}\left[i+m_{1}\right], \ldots, A^{\prime}\left[i+m_{l}\right]\right)$.

- Locate $f(i)$ in the search tree, creating a new leaf if necessary.

- Add $i$ to the $A$-list for $f(i)$.

5. For $j=1,2, \ldots, g$ do

- Compute fingerprint $f(j)=\left(I\left[j+m_{1}\right], \ldots, I\left[j+m_{l}\right]\right)$.

- Locate $f(j)$ in the search tree, creating a new leaf if necessary.

- Add $j$ to the $B$-list for $f(j)$.

6. Quantizing: For each fingerprint $f$, scan the $B$-list for $f$ and round each $j$ value to the nearest multiple of $Q / 4$, deleting repeated values; also scan the $A$-list for $f$ and round each $i$ value to the nearest multiple of $Q / 4$, deleting repeated values.

7. For each fingerprint $f$, each (rounded) $i^{\prime}$ in $f^{\prime}$ 's $A[Q / 4]$ list, and each (rounded) $j^{\prime}$ in $f^{\prime}$ s $B[Q / 4]$ list, output $t=\left(j^{\prime}-i^{\prime}\right)[Q]$ (that is, $\left(j^{\prime}-i^{\prime}\right)$ rounded to the nearest multiple of $Q$ ).

Figure 2: "Ruler" procedure for finding approximate block shifts.

$\operatorname{DECIDE}(A, B, \alpha, C)$

0 . Choose sufficiently small $\epsilon$, and sufficiently large $c_{1}$ (for the given $\alpha$ and $C$ ).

1. Let quantization parameter $Q=\epsilon \cdot \min \left\{n^{1-\alpha}, n^{\alpha / 2}\right\}$.

2. Set $T=\operatorname{Matches}\left(A, B, n^{\alpha}\right)$.

3. If $T$ is nonempty, then output $C L O S E$, else output FAR.

$\operatorname{matches}(A, I, E)$

1. If $E<1$, use SHIFTs to compute $T$.

2. If $E \geq 1$,

2a. Set $k=\min \left\{\epsilon n^{1-\alpha}, 2 c_{1} E\right\}$.

2b. Decompose $I$ into a set $\mathcal{I}$ of contiguous disjoint intervals of size $|I| / k$.

2c. Compute $T=\operatorname{Coord-matches}\left(A, \mathcal{I}, E, c_{1} E / k, k / c_{1}\right)$.

3. Return $T$.

Figure 3: The Edit Distance Testing Algorithm 
Lemma 6. If $\mathcal{D}(A, B) \leq n^{\alpha}$, then the algorithm outputs CLOSE with high probability, for any parameter values $\epsilon<1$ and $c_{1}>1$.

Proof. (Sketch) Note that it is sufficient to prove that if there is a (quantized) $(t, E)$-matching of $B$ with respect to $A$, the top-level invocation of MATCHES will find it, with high probability. We prove this statement by induction on the height $h$ of the invocation of MATCHES. For $h=0$, this is true because of the correctness of the SHIFTs procedure. Assuming it is true for height $(h-1)$, we show that it is true for height $h$ as well: Let MATCHES $(A, I, E)$ be a height $h$ invocation, and suppose that $I$ has a $(t, E)$-matching with respect to $A$. Then, by Lemma 2 , there exists a coordinated matching with the parameters specified in Step (2c) of MATCHES. Then, using a variant of Lemma 4 , we see that most of the sampled intervals have some match with translation $t_{i}$ close to $t$. Now, consider the recursive calls to MATCHES made by COORD-MATCHES. By the inductive assumption, they will report these matches $t_{i}$ with high probability. Now, using Lemma 5, we know that they will find these translations $t_{i}$, and hence COORD-MATCHES will report $t$ among its output $T$. Hence, the translation $t$ is the output of the level $h$ MATCHES, with high probability.

It remains to show that the algorithm outputs $F A R$ with high probability when $\mathcal{D}(A, B)>n / C$, for an appropriate choice of constants.

LEMma 7. There exist values for the constants $\epsilon$ and $c_{1}$ (dependent only on $\alpha$ and $C$ ), such that with high probability, if the algorithm outputs CLOSE then $\mathcal{D}(A, B)<n / C$.

Proof. (Sketch) First, as the quantization is at most $\epsilon$ times the size of the smallest interval size in the recursion, it alters the constants but not the qualitative matching results (see Proposition 1). Second, as long as the underlying SHIFTs algorithm and interval sampling procedures take sufficiently many samples $(O(\log n))$, the effects of their imprecision can be reduced to $\epsilon$ amounts as well. For the rest of the discussion, we ignore these issues.

The other potential source of error is in inferring the existence of a matching from a coordinated matching, at each level of the recursion. We use Lemma 1 to bound this error; this involves a careful analysis of the $\beta$ factors that arise at each level of the recursion.

We consider the values of $S^{\prime}, \sigma, E$, and $(D / k)$ in each invocation of COORD-MATCHES. Let $\lambda=\epsilon \cdot n^{1-\alpha}$. In each of the first $r-2$ levels, MATCHES subdivides the interval into $\lambda$ intervals. At height $r$ (the top level), we have

$$
S^{\prime}=\frac{n}{\lambda}, \sigma=n^{\alpha}, E=n^{\alpha} \cdot \frac{c_{1}}{\lambda},(D / k)=\frac{1}{c_{1}}
$$

Now, let us write $\beta_{h}$ for the factor $\beta$ in Lemma 1, corresponding to the values of $S^{\prime}, \sigma, E$, and $(D / k)$ at height $h$. We have

$$
\beta_{r}=\frac{2 n^{\alpha} \lambda}{n}+\epsilon+\frac{1}{c_{1}}=3 \epsilon+\frac{1}{c_{1}}
$$

Proceeding in this manner, we find at level 2,

$$
\begin{gathered}
S^{\prime}=\frac{n}{\lambda^{r-1}}, \sigma=n^{\alpha} \cdot\left(\frac{c_{1}}{\lambda}\right)^{r-2}, E=n^{\alpha} \cdot\left(\frac{c_{1}}{\lambda}\right)^{r-1},(D / k)=\frac{1}{c_{1}} \\
\beta_{2}=\frac{2 n^{\alpha} c_{1}^{r-2} \lambda}{n}+\epsilon+\frac{1}{c_{1}} \\
=2 c_{1}^{r-2} \cdot \epsilon+\epsilon+\frac{1}{c_{1}}
\end{gathered}
$$

At this level, we must have $2 c_{1} E<\lambda$, or else the recursion would not terminate at level $r$. Hence, the pattern changes here. However, since $\sigma$ reduces by a factor of $\lambda / c_{1}$ and $S^{\prime}$ reduces by a factor $2 c_{1} E<\lambda$, we have that $\sigma / S^{\prime}$ can increase by at most another factor of $c_{1}$, and so we have

$$
\beta_{1} \leq 2 c_{1}^{r-1} \cdot \epsilon+\epsilon+\frac{1}{c_{1}}
$$

Note that as $r$ is fixed (by $\alpha$ ), we can pick values of $c_{1}$ and $\epsilon$ to achieve $\beta_{1}$ as small as we wish: we first select a suitable value of $c_{1}$, and then select $\epsilon$ based on the chosen $c_{1}$. In particular, we can pick values such that $\beta_{1}<\frac{1}{r C}$. Further, observe that $\beta_{r}, \ldots, \beta_{2}<\beta_{1}$.

Now, suppose algorithm DECIDE outputs CLOSE. This means that at each level of the recursion, we have a coordinated matching. Consider an instance of COORD-MATCHES at height 1 , and apply Lemma 1 . This says that the corresponding interval at height 2 has a matching with additional edit distance at most $\beta_{1}$ times the length of the interval. Adding up the edits over all intervals in a level, and over all levels of recursion, we see that the total edit distance of $A$ from $B$ is less than $\beta_{1} n . r<\frac{n}{C}$.

Combining Lemma 6 and 7, we get the following theorem:

Theorem 2. For any fixed $\alpha<1$, we can choose constants $\epsilon$ and $c_{1}$ such that procedure DECIDE solves the edit distance testing problem with high probability.

\subsection{Running time analysis}

In this section, we provide the running time analysis of our algorithm. The analysis is based on three cases, depending on the value of $\alpha$.

\section{Case (i): $\alpha<1 / 2$}

In this case, there will only be one level of recursion. At the top level, $B$ will be broken into intervals of size $O\left(n^{\alpha}\right)$; the expected number of edits per interval is less than 1 , and so in the next level the SHIFTs procedure will be used to find the matches of these intervals. Thus, there are $d \log n$ calls to SHIFTs; for the specified $Q$, each call takes $O\left(n^{\alpha / 2} \log n\right)$ time. In addition, there is one call to QMERGE, which takes $O\left(n^{\alpha / 2} \log n\right)$ time, thus giving us a total running time of $O\left(n^{\alpha / 2} \log ^{2} n\right)$.

\section{Case (ii): $1 / 2<\alpha<2 / 3$}

When $1 / 2<\alpha<2 / 3$, there will be two levels of recursion. At the top level, we break $B$ into intervals of size $c_{1} n^{\alpha}$. In the second level, each selected interval is further broken into subintervals of size $n^{\alpha / 2}$. Finally, we find matches for these subintervals using shifTs. Thus, there are $O\left(\log ^{2} n\right)$ calls to SHIFTs; again, each call takes $O\left(n^{\alpha / 2} \log n\right)$ time. There are also $O(\log n)$ calls to QMERGE. All together, the running time is $O\left(n^{\alpha / 2} \log ^{3} n\right)$.

\section{Case (iii): $\alpha>2 / 3$}

We now consider the general case, when there are $r>2$ levels of recursion. We point out that there are two sources of degradation in this recursive algorithm. First, we incur a time and query overhead of $(\log n)^{r+O(1)}$ because of the random sampling at each level of recursion. The second degradation, which is more significant, comes from the SHIFTS procedure at the final stage of the recursion. Each invocation of SHIFTs has to find all (quantized) shifts in the 
range $\left[-n^{\alpha} \ldots n^{\alpha}\right]$ of a block of size $O\left(n^{1-\alpha}\right)$. However, for $\alpha>2 / 3$, we have $n^{1-\alpha}<\sqrt{n^{\alpha}}$, and so the ruler used in the SHIFTS procedure has to be asymmetric. As a result, the running time of each invocation is $O\left(n^{2 \alpha-1} \log n\right)$. Thus, the edit distance testing algorithm has an overall running time of $\tilde{\mathrm{O}}\left(n^{2 \alpha-1}\right)$.

\section{A LOWER BOUND ON QUERY COM- PLEXITY}

This section proves a query-complexity lower bound for the edit distance problem defined in Section 2:

TheOREM 3. Any probabilistic algorithm for the edit distance problem requires $\Omega\left(n^{\alpha / 2}\right)$ queries.

In fact, we show a $\Omega\left(n^{\alpha / 2}\right)$ lower bound for the possibly easier problem of distinguishing a pair $(A, B)$ of random strings from a pair $(A, B)$ where $A$ is random and $B$ is a right shift of $A$ by $t$ positions for a random $t \in\left[n^{\alpha} / 2, n^{\alpha}\right]$. Since two random strings have a linear edit distance, Theorem 3 follows.

LeMma 8. With probability at least $1-o(1)$, two random $n$-bit strings have edit distance $\geq n / 6$.

Proof. It is enough to show that for a fixed $n$-bit string $X$, the fraction of strings within edit distance $n / 6$ of $X$ is $o(1)$. A string that is at most $d$ away from $X$ is obtained by choosing $d$ locations, and for each of the locations, picking one for the deletion, replacement, insertion of a new bit, or no-edit operation. Thus, the number of strings within edit distance $d$ from $X$ is at most $\left(\begin{array}{l}n \\ d\end{array}\right) \cdot 5^{d}$. Substituting $d=n / 6$ and using the fact that $\left(\begin{array}{c}n \\ \beta n\end{array}\right) \leq 2^{H_{2}(\beta) n}$ where $H_{2}(p)$ is the binary entropy function $-p \log p-(1-p) \log (1-p)$, we get that the number of strings obtained from $X$ with at most $n / 6$ edit operations is at most

$$
\left(\begin{array}{c}
n \\
n / 6
\end{array}\right) 5^{n / 6} \leq 2^{\left(H_{2}(1 / 6)+1 / 2\right) n}=o\left(2^{n}\right) .
$$

We define two distributions $\mathcal{F}$ and $\mathcal{C}$ on pairs of strings. Let $\mathcal{F}$ be a distribution on pairs of random $n$-bit strings. Let $\mathcal{C}$ be a distribution on pairs of $n$-bit strings $(A, B)$ where $A$ is random, and $B$ is obtained from $A$ by appending a $t$-bit random string at the beginning and deleting $t$ bits at the end, for a randomly chosen $t \in\left[n^{\alpha} / 2, n^{\alpha}\right]$. By Lemma 8, with high probability, $\mathcal{F}$ produces a pair of strings with edit distance at least $n / 6$ while $\mathcal{C}$ is over pairs of strings with edit distance at most $n^{\alpha}$. The following lemma together with Lemma 8 and Yao's minmax principle proves Theorem 3.

Lemma 9. Let $q<n^{\alpha / 2}$. Then for every q-query deterministic algorithm $\mathcal{A}$,

$$
\left|\operatorname{Pr}_{x \leftarrow \mathcal{F}}[\mathcal{A}(x)=1]-\operatorname{Pr}_{x \leftarrow \mathcal{C}}[\mathcal{A}(x)=1]\right| \leq \frac{1}{2} .
$$

Proof. Consider the event $R$ when the input to $\mathcal{A}$ comes from the distribution $\mathcal{F}$ and the queries of $\mathcal{A}$ reveal the shift $t$ between the two strings, namely $\mathcal{A}$ queries a location $i$ in $A$ and $i+t$ in $B$. Notice that when event $R$ does not happen, all the bits $\mathcal{A}$ sees are random and so it cannot possibly distinguish between the distributions $\mathcal{C}$ and $\mathcal{F}$. Therefore

$$
\left|\operatorname{Pr}_{x \leftarrow \mathcal{F}}[\mathcal{A}(x)=1]-\operatorname{Pr}_{x \leftarrow \mathcal{C}}[\mathcal{A}(x)=1]\right| \leq \operatorname{Pr}[R] .
$$

Similarly, as long as a shift of $t$ was not revealed by $\mathcal{A}$, the bits it sees are random; when such a shift is revealed, event $R$ occurs. This means that the probability of $R$ is unaltered when we deprive $\mathcal{A}$ of its adaptive behaviour. We therefore assume that $\mathcal{A}$ queries $q$ locations (in either strings) at once. Such a selection reveals at most $(q / 2)^{2}$ shifts and now, since $t$ is randomly chosen among $n^{\alpha} / 2$ values, we have that

$$
\operatorname{Pr}[R] \leq \frac{(q / 2)^{2}}{n^{\alpha} / 2} \leq 1 / 2 .
$$

\section{REFERENCES}

[1] W. Chang and E. Lawler. Approximate string matching in sublinear expected time. In Proceedings of the 31st IEEE Annual Symposium on Foundations of Computer Science, pages 116-124, Saint Louis, Missouri, 1990. IEEE Computer Society Press.

[2] R. Cole and R. Hariharan. Approximate string matching: A simpler faster algorithm. In Proceedings of the Ninth Annual ACM-SIAM Symposium on Discrete Algorithms, pages 463-472, San Francisco, California, Jan. 1998.

[3] G. Cormode and S. Muthukrishnan and C. Sahinalp and U. Vishkin. Communication complexity of document exchange. In Proceedings of the Eleventh Annual ACM/SIAM Symposium on Discrete Algorithms, pages 197-206, San Francisco, CA, Jan. 2000.

[4] G. M. Landau and U. Vishkin. Introducing efficient parallelism into approximate string matching and a new serial algorithm. In Proceedings of the Eighteenth annual ACM Symposium on Theory of Computing, pages 220-230, Berkeley, California, May 1986. ACM Press, New York.

[5] W. J. Masek and M. S. Paterson. A faster algorithm computing string edit distances. Journal of Computer and System Sciences, 20:18-31, 1980.

[6] E. W. Myers. A sublinear algorithm for approximate keyword searching. Algorithmica, 12(4/5):345-374, Oct./Nov. 1994.

[7] S. C. Sahinalp and U. Vishkin. Efficient approximate and dynamic matching of patterns using a labeling paradigm. In 37th Annual Symposium on Foundations of Computer Science, pages 320-328, Burlington, Vermont, Oct. 1996. IEEE Computer Society Press. 